\title{
On the leading OPE corrections to the ghost-gluon vertex and the Taylor theorem
}

\author{
Ph. Boucaud, ${ }^{a}$ D. Dudal, ${ }^{b}$ J.P. Leroy, ${ }^{a}$ O. Pène ${ }^{a}$ and J. Rodríguez-Quintero ${ }^{c}$ \\ ${ }^{a}$ Laboratoire de Physique Théorique, Université de Paris XI, \\ Bâtiment 211, 91405 Orsay Cedex, France \\ ${ }^{b}$ Department of Physics and Astronomy, Ghent University, \\ Krijgslaan 281 S9 9000 Gent, Belgium \\ ${ }^{c}$ Dpto. Fisica Aplicada, Fac. Ciencias Experimentales, Universidad de Huelva, \\ Avda. Fuerzas Armadas, s.n.; 21071 Huelva, Spain \\ E-mail: philippe.boucaud@th.u-psud.fr, david.dudal@ugent.be, \\ jean-pierre.Leroy@th.u-psud.fr, olivier.pene@th.u-psud.fr, \\ jose.rodriguez@dfaie.uhu.es
}

ABSTRACT: This brief note is devoted to a study of genuine non-perturbative corrections to the Landau gauge ghost-gluon vertex in terms of the non-vanishing dimension-two gluon condensate. We prove these corrections to give account of current SU(2) lattice data for the vertex with different kinematical configurations in the domain of intermediate momenta, roughly above $2-3 \mathrm{GeV}$. We pay special attention to the kinematical limit which the bare vertex takes for its tree-level expression at any perturbative order, according to the wellknown Taylor theorem. Based on our OPE analysis, we also present a simple model for the vertex, in acceptable agreement with the lattice data also in the IR domain.

KeYwords: Nonperturbative Effects, Sum Rules, QCD, Lattice QCD

ARXIV EPRINT: 1109.3803 


\section{Contents}

1 Introduction 1

2 The OPE for the ghost-gluon vertex 3

3 A model for the Euclidean ghost-gluon vertex 5

3.1 The model 5

$\begin{array}{ll}3.2 & \text { Comparing with available lattice data } \\ \end{array}$

4 The Taylor kinematics and the asymmetric gluon-ghost vertex 9

5 Conclusions 12

\section{Introduction}

The infrared properties of the Landau-gauge QCD Green functions have motivated many studies in the last few years, mainly involving both lattice (see for instance refs. [1-8]) and continuum approaches (see for instance refs. [9-17] using Dyson-Schwinger equations (DSE), [18-21] using the refined Gribov-Zwanziger formalism or [22, 23] using the CurciFerrari model as an effective description). Most of the DSE analysis take advantage of approximating the ghost-gluon vertex by a constant when truncating the infinite tower of the relevant equations. To be more precise, only the behaviour of the involved transverse form factor needs to be approximated by a constant for the purpose of truncating the ghost propagator DSE (see also the recent paper [24]). However, this ghost propagator DSE (GPDSE) appears to be a cornerstone for the analysis of infrared DSE solutions for the gluon correlations functions: the two classes of solutions, namely "decoupling" and "scaling", depend on the regular or singular behaviour of the ghost dressing function at zero-momentum and on how this constrains the gluon behaviour via the GPDSE [11]. Most of the ammo for the approximation of the ghost-gluon vertex and GPDSE truncation is mainly provided by the Taylor theorem which is widely known as a non-renormalization one [25]. This claims that, in the particular kinematical configurations defined by a vanishing incoming ghost-momentum, no non-zero radiative correction survives for the Landaugauge ghost-gluon vertex, which takes thus its tree-level expression at any perturbative order [25]. This statement entails two important consequences: that (i) the bare ghostgluon vertex is UV-finite for any kinematical configuration, and that (ii) in the specific MOM scheme where the renormalization point is taken with a vanishing incoming ghost 
momentum (which we named the Taylor scheme — T-scheme — in [26]) the renormalization constant for the ghost-gluon vertex ${ }^{1}$ is exactly 1 . Then, one can use those two conclusions to write (schematically) the renormalized GPDSE as

$$
\frac{1}{F\left(k^{2}\right)}=1+\alpha_{T}\left(\mu^{2}\right) \int d^{4} q K(k, q) H_{1}^{\text {bare }}(q, k) F\left(q^{2}\right),
$$

where $\mu$ is the subtraction momentum for all the renormalized quantities, $F$ is the ghostpropagator dressing function, $K(k, q)$ is the part of the kernel which does not involve the ghost-gluon vertex, $\alpha_{T}$ is the running coupling in the T-scheme, ${ }^{2}$ and $H_{1}^{\text {bare }}$ is the transverse form factor of the finite bare ghost-gluon vertex, defined by

$$
\Gamma_{\mu}^{a b c}(-q, k ; q-k)=-g_{0} f^{a b c}\left(q_{\mu} H_{1}(q, k)+(q-k)_{\mu} H_{2}(q, k)\right) .
$$

The kernel $K$ corresponds to the gluon propagator, its transverse character in the Landau gauge allows to project out the $H_{2}$-form factor from expression (1.2). Therefore, we have chosen to call the surviving piece, i.e. $H_{1}$, the transverse form factor. Extending this nomenclature, we call $\mathrm{H}_{2}$ the longitudinal vertex form factor. The kernel $\mathrm{K}$ can be either built by invoking lattice data (as done, for instance, in ref. [12]) or determined by solving the coupled system of eq. (1.1) and the gluon propagator DSE. In both cases, a precise determination of the ghost dressing function from eq. (1.1), to be confronted for instance with lattice data, requires a correspondingly precise knowledge of the transverse form factor $H_{1}$. Thus, the ghost-gluon vertex plays an important quantitative and qualitative rôle for the infrared DSE analysis of Yang-Mills QCD Green functions. In fact, with the usual bare ghost-gluon vertex approximation, namely that the bare transverse form factor is 1 over all the momentum range, it is readily appreciated from e.g. the results of $[12,30]$ that when the lattice data for the gluon propagator is fed into the GPDSE, there is a qualitative difference with the ghost propagator lattice data, unless one boosts by hand the input for the coupling constant at the renormalization point to an artificially large value. Another way-out [12] is to impose the bare transverse form factor to take a constant, clearly different from 1 , to borrow the boost of the coupling input needed to describe lattice data. ${ }^{3}$ This is strongly hinting towards a missing piece in the ghost-gluon vertex, which, if stronger than a tree level one, will lift the output of the GPDSE up to the ghost lattice data (see refs. [31, 32]). Of course, before this can be precisely tested in practice, we need to cook up a reliable input for the ghost-gluon vertex. How to do this will be investigated in this work.

\footnotetext{
${ }^{1}$ It can also be straightforwardly concluded that the ghost-gluon vertex renormalization constant, $\widetilde{Z}_{1}$, is exactly 1 in the $\overline{\mathrm{MS}}$ scheme.

${ }^{2}$ The coupling in T-scheme has been profusely computed on the lattice and confronted to perturbative predictions to estimate $\Lambda_{\overline{\mathrm{MS}}}[26-28]$. It has been also used to define an effective charge that could be applied for phenomenological purposes [29].

${ }^{3}$ As it is shown in refs. [31, 32], taking a constant larger than 1 for the bare $H_{1}$ helps to account better, but not enough, for the ghost propagator lattice data with the results of the integration of the GPDSE. Some non-perturbative structure for the ghost-gluon vertex is thus needed, and it is also preliminary shown there that the sort of inputs we can obtain with the OPE analysis in this work might very well lead to a good description of lattice data.
} 
On the other hand, the study of the running of the QCD Green functions triggered some lattice works [33-44], many of them aiming to determining the $\Lambda_{\mathrm{QCD}}$ parameter, which concluded that the impact of the non-perturbative corrections coming from the dimension-two gluon condensate $\left\langle A^{2}\right\rangle$ was not negligible [37-41]. In particular, in ref. [26] we computed the running coupling renormalized in the Taylor scheme from the lattice in pure Yang-Mills gauge theory and proved the necessity of including non-perturbative power corrections to describe properly the running over a momenta window from around 3 to $5 \mathrm{GeV}$. In the T-scheme, the configuration of momenta for the ghost-gluon vertex is the one "protected" by the non-renormalization theorem and, at least in perturbation theory to any order, no correction to the bare ghost-gluon vertex is expected beyond the tree-level expression. However, non-perturbative OPE corrections should be expected for any other kinematical configuration and, furthermore, it is interesting to examine whether a genuine non-perturbative correction might survive in the particular configuration where Taylor's argument works. More interesting will be to investigate whether, for the same price, these non-perturbative corrections related to $\left\langle A^{2}\right\rangle$ might suggest, apart from describing the current lattice data inside the appropriate momenta window, a reliable input for the ghost-gluon vertex we mentioned above. This is the main purpose of this note.

\section{The OPE for the ghost-gluon vertex}

The OPE procedure for the Landau-gauge ghost-gluon vertex,

$$
\begin{aligned}
V_{\mu}^{a b c}(-q, k ; q-k) & =\Gamma_{\mu^{\prime}}^{a^{\prime} b^{\prime} c^{\prime}}(-q, k ; q-k) G_{\mu \mu^{\prime}}^{b b^{\prime}}(q-k) F^{a a^{\prime}}(q) F^{c c^{\prime}}(k) \\
& =\int d^{4} y d^{4} x e^{i(q-k) \cdot x} e^{i k \cdot y}\left\langle T\left(c^{c}(y) A_{\mu}^{b}(x) \bar{c}^{a}(0)\right)\right\rangle
\end{aligned}
$$

is quite similar to the one described in ref. [26]. Here, the OPE expansion shall read

$$
\begin{aligned}
V_{\mu}^{a b c}(-q, k ; q-k)= & \left(d_{0}\right)_{\mu}^{a b c}(q, k) \\
& +\left(d_{2}\right)_{\mu a^{\prime} b^{\prime}}^{a b \mu^{\prime} \nu^{\prime}}(q, k)\left\langle: A_{\mu^{\prime}}^{a^{\prime}}(0) A_{\nu^{\prime}}^{b^{\prime}}(0):\right\rangle+\cdots
\end{aligned}
$$

where $d_{0}$ accounts for the purely perturbative contribution to the vertex, while

$$
\begin{aligned}
w_{\mu}^{a b c} & =\left(d_{2}\right)_{\mu a^{\prime} b^{\prime}}^{a b c \mu^{\prime} \nu^{\prime}}(q, k) \delta^{a^{\prime} b^{\prime}} g_{\mu^{\prime} \nu^{\prime}} \\
& =2 I^{[1]}+2 I_{s}^{[1]}+2 I^{[2]}+4 I^{[3]}+2 I^{[4]}+I^{[5]}
\end{aligned}
$$

where $^{4}$

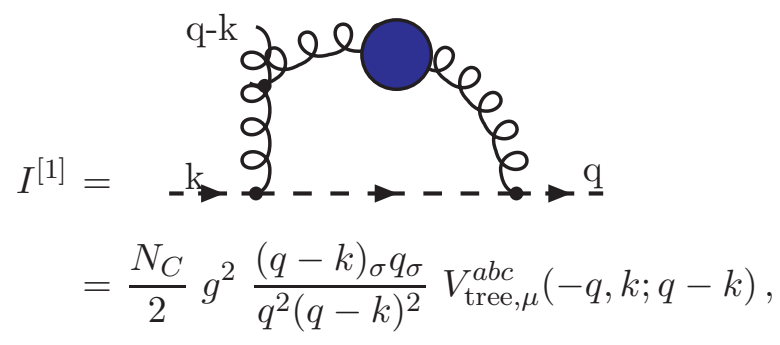

\footnotetext{
${ }^{4} N_{C}$ is the number of colours.
} 
the tree-level ghost-gluon vertex being

$$
V_{\text {tree }, \mu}^{a b c}(-q, k ; q-k)=-i \frac{g}{k^{2} q^{2}(q-k)^{2}} f^{a b c} q_{\mu_{2}} g_{\mu_{2} \mu}^{\perp}(q-k),
$$

while

$$
\begin{aligned}
I_{s}^{[1]} & =-12 \varepsilon_{-1}^{\mathrm{q}-\mathrm{k}} \\
& =-\frac{N_{C}}{2} g^{2} \frac{(q-k)_{\sigma} k_{\sigma}}{k^{2}(q-k)^{2}} V_{\mathrm{tree}, \mu}^{a b c}(-q, k ; q-k),
\end{aligned}
$$

and

$$
\begin{aligned}
& = \\
& =\frac{N_{C}}{4} g^{2} \frac{k_{\sigma} q_{\sigma}}{q^{2} k^{2}} V_{\text {tree }, \mu}^{a b c}(-q, k ; q-k) .
\end{aligned}
$$

The other contributions, $I^{[3,4,5]}$, can be immediately written using the previous results:

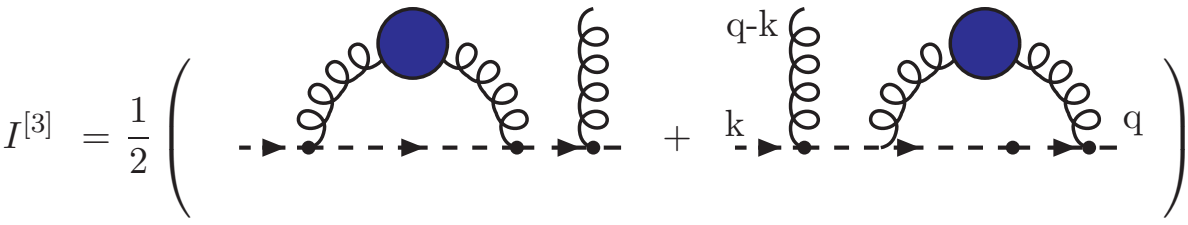

$$
\begin{aligned}
& =\left(\frac{N_{C}}{4} \frac{g^{2}}{k^{2}}+\frac{N_{C}}{4} \frac{g^{2}}{q^{2}}\right) V_{\text {tree }, \mu}^{a b c}(-q, k ; q-k)
\end{aligned}
$$

and

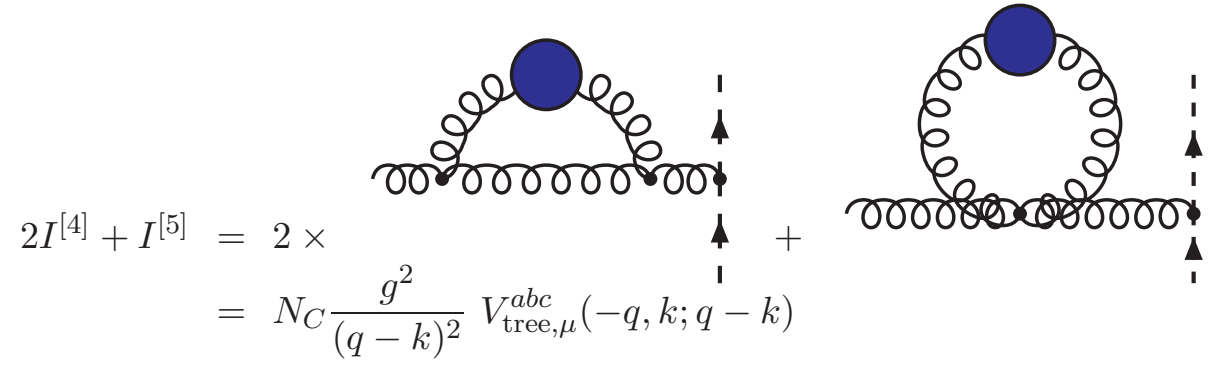

In all cases, the blue bubble refers to a contraction of the colour and Lorentz indices with $\frac{1}{2} \delta_{a b} g_{\mu \nu}$. We have also introduced the notation $g_{\mu \nu}^{\perp}(\ell)=g_{\mu \nu}-\frac{\ell_{\mu} \ell_{\nu}}{\ell^{2}}$ for the transverse projector.

Then, one obtains

$$
w_{\mu}^{a b c}=g^{2}\left(s_{V}(q, k)+s_{F}(k)+s_{F}(q)+s_{G}(k-q)\right) V_{\text {tree }, \mu}^{a b c}(-q, k ; q-k)
$$


with

$$
\begin{aligned}
s_{G}(q) & =s_{F}(q)=\frac{N_{C}}{q^{2}}, \\
s_{V}(q, k) & =\frac{N_{C}}{2}\left(2 \frac{(q-k) \cdot q}{q^{2}(q-k)^{2}}+2 \frac{(k-q) \cdot k}{k^{2}(q-k)^{2}}+\frac{k \cdot q}{k^{2} q^{2}}\right) .
\end{aligned}
$$

$s_{F}$ (resp. $s_{G}$ ) comes from the OPE corrections to the external ghost (resp. gluon) propagator, i.e. from the non-proper diagrams in eqs. (2.8), (2.9) and $s_{V}$ from the proper vertex correction in eqs. (2.4), (2.7). The OPE corrections to the proper vertex can be obtained from there, remembering that the bare ghost-gluon vertex reads as:

$$
\Gamma_{\mu}^{a b c}(-q, k ; q-k)=-g_{0} f^{a b c}\left(q_{\mu} H_{1}(q, k)+(q-k)_{\mu} H_{2}(q, k)\right) .
$$

Then, in Landau gauge, only the form factor $H_{1}$ survives in the Green function to give:

$$
V_{\mu}^{a b c}(-q, k ; q-k)=-i g_{0} f^{a b c} q_{\mu^{\prime}} g_{\mu^{\prime} \mu}^{\perp}(q-k) H_{1}(q, k) G\left((q-k)^{2}\right) F\left(q^{2}\right) F\left(k^{2}\right),
$$

where $G$ and $F$ are the gluon and ghost dressing functions for which we previously computed the non-perturbative OPE corrections. Thus, one would have:

$$
H_{1}(q, k)=H_{1}^{\text {pert }}(q, k)\left(1+s_{V}(q, k) \frac{\left\langle A^{2}\right\rangle}{4\left(N_{C}^{2}-1\right)}+\mathcal{O}\left(g^{4}, q^{-4}, k^{-4}, q^{-2} k^{-2}\right)\right) .
$$

All the non-proper corrections have been removed in the usual way.

\section{A model for the Euclidean ghost-gluon vertex}

We will now devote this section to model the transverse form factor of the Euclidean ghostgluon vertex, $H_{1}$, on the ground provided by eq. (2.14), and compare the result with some recent lattice data for this form factor computed in different kinematical configurations.

\subsection{The model}

In Euclidean metrics the bare ghost-gluon vertex can be written very similarly as:

$$
\Gamma_{\text {bare }, \mu}^{a b c}(-q, k ; q-k)=i g_{0} f^{a b c}\left(q_{\mu} H_{1}(q, k)+(q-k)_{\mu} H_{2}(q, k)\right),
$$

where the form factor $H_{1}$ plays a crucial rôle when solving the ghost-propagator DysonSchwinger equation (GPDSE) as discussed above. The OPE non-perturbative corrections to the form factor $H_{1}$ were obtained in eq. (2.14), but that result is in principle only reliable for large enough $k, q$ and $q-k$ since the SVZ factorization on which it relies might not be valid for low momenta. Nevertheless, in the following we will propose a very simple conjecture to extend eq. (2.14) to any momenta, which will provide us with a calculational model for the ghost-gluon vertex to continue research with. In particular, the perturbative 
part of the ghost-gluon vertex is usually approximated by a constant behaviour; ${ }^{5}$ if we then apply a finite renormalization prescription such that

$$
\left.\widetilde{Z}_{1}\left(\mu^{2}\right) H_{1}(q, k)\right|_{\mu^{2}}=1
$$

where the renormalization momentum, $\mu^{2}$, for a given kinematical configuration (for instance, $q-k=0$ and $q^{2}=k^{2}=\mu^{2}$ ) is chosen to be large enough, on the basis of eq. (2.14), we can conjecture that

$$
\begin{aligned}
H_{1}\left(q^{2}, k^{2}, \theta\right)=\widetilde{Z}_{1}^{-1}\left[\begin{array}{l}
1+ \\
+\frac{N_{C} g^{2}\left\langle A^{2}\right\rangle}{8\left(N_{C}^{2}-1\right)}
\end{array}\right. \\
\times\left(\frac{\sqrt{k^{2} q^{2}} \cos \theta}{k^{2} q^{2}+m_{\mathrm{IR}}^{4}}+2 \frac{q^{2}-\sqrt{k^{2} q^{2}} \cos \theta}{q^{2}\left(q^{2}+k^{2}-2 \sqrt{q^{2} k^{2}} \cos \theta\right)+m_{\mathrm{IR}}^{4}}\right. \\
\left.\left.\quad+2 \frac{k^{2}-\sqrt{k^{2} q^{2}} \cos \theta}{k^{2}\left(q^{2}+k^{2}-2 \sqrt{q^{2} k^{2}} \cos \theta\right)+m_{\mathrm{IR}}^{4}}\right)\right],
\end{aligned}
$$

gives a reasonable description of the ghost-gluon form factor $H_{1}$ all over the range of its momenta $q$ and $k$, where $\theta$ stands for the angle between them. The purpose of eq. (3.3) is to keep the main features of the momentum behaviour of the ghost-gluon form factor provided by the OPE analysis and to give a rough description of the deep IR only with the introduction of some IR mass scale, $m_{\mathrm{IR}}$, which is mainly aimed to avoid the spurious singularities resulting from the OPE expansion in momentum inverse powers.

It should be noted that the OPE technology implies first a hierarchic expansion in terms of local operators and next a factorization assumption that allows for all the largedistance information to be encoded in the condensates of those local operators in the QCD vacuum. If the momentum to be described decreases, more condensates of higher and higher dimensions should be needed, but also the Wilson coefficient containing the shortdistance information should be computed at a higher loop level. To try to sum up all this is, if not impossible, a daunting task. This is why the OPE is applied to provide only an asymptotic expansion which is reliable to account for some QCD phenomenology, but nevertheless it can also successfully reproduce quenched and unquenched lattice results for many Green functions in some intermediate momentum region [26, 39-41]. Then, it is important to stress here that the model vertex (3.3) will never be obtainable from the OPE in QCD, as it is just impossible to describe with the OPE whatever quantity over a full momentum range. Very recently, a Landau gauge version of the Curci-Ferrari model has been shown to give, at the first perturbative order, a reliable description of quenched QCD lattice results $[22,23]$. There is no reason to think that the same approach could also account for the operator expansion and factorization sum rules. As a matter of fact,

\footnotetext{
${ }^{5}$ At least for large momenta, this seems to be the case in lattice simulations [45, 46] for several kinematical configurations, and so is confirmed also by the perturbative calculations in refs. [47, 48].
} 
one would obtain that applying the OPE technology with a massive gluon propagator, for instance with a frozen mass $M$, implies to replace

$$
\frac{1}{(q-k)^{2}} \rightarrow \frac{1}{(q-k)^{2}+M^{2}}
$$

in eqs. (2.11), (2.14) and this does not totally cure the above mentioned spurious singularities coming from the expansion in momentum inverse powers. In fact, assuming that $M$ is the typical mass scale of a theory, the OPE machinery only intends to describe physics for momenta (much) larger than $M$. However, lattice results, as can be seen in the next subsection, and the Taylor theorem in its particular limit prove that no singularity appear in the IR. This is why we introduce in eq. (3.3) the mass scale, $m_{\mathrm{IR}}$, that can be thought as some sort of IR regulator that should be related to some kind of effective gluon mass scale.

The proposed expression is thus supposed to describe the vertex for the whole range of possible momenta, as needed to be plugged into the GPDSE in future work, while any OPE analysis, from its very definition, can only be valid for relatively large momenta. The proposal (3.3) has however the right asymptotic momentum behaviour, as compared to the OPE result (2.14). Its validity for lower momenta will shortly be checked by a confrontation with the available lattice data in the next subsection. Said otherwise, eq. (3.3) can be also seen as a parametrization to describe lattice data (to be properly plugged into the GPDSE) that, behaving asymptotically as the OPE predicts, benefits of the fact that free parameters as $\left\langle A^{2}\right\rangle$ can be borrowed from (or compared with) different and independent analyses. It is anyhow very important to stress that it is the OPE analysis leading to (2.14) providing us with the kinematical structure of (3.3), at least in the intermediate region, and that the infrared completion has nothing to do with this. Thus, the success in the confrontation with the available lattice data, with different kinematical combinations for the form factor, will also confirm that the OPE analysis works in the appropriate region and it also gives an independent estimate of $\left\langle A^{2}\right\rangle$.

Furthermore, to give further credibility to the vertex (3.3), we also plan a future study of the ghost-gluon vertex from the analytical viewpoint. For example, it would be instructive to find out if the refined Gribov-Zwanziger (RGZ) computational scheme could give access to a vertex of the type modeled here, since this formalism incorporates effects of nonperturbative dimension 2 condensates in a controllable, renormalizable setting, applicable to the whole range of momenta. It is worth to remark that, as an enlightening indication, the gluon propagator in the RGZ scheme can be asymptotically expanded to agree with its OPE prediction stemming from the gluon condensate, $\left\langle A^{2}\right\rangle$; however, the deep infrared structure of the propagator clearly escapes from the OPE analysis and depends, for instance, on additional dimension two-condensates and on the Gribov-Zwanziger parameter defined through the so-called Gribov horizon condition [18-21]. An alternative could be the above-mentioned Landau gauge version of the Curci-Ferrari model, which was already tested at the propagator level in $[22,23]$ but that can be also applied to give a prediction for the ghost-gluon vertex.

Of course, $\mu^{2}$ being large enough, $\widetilde{Z}_{1}$ can be approximated by some constant value for any $\mu^{2}$ because the logarithmic behaviour of the ghost-gluon in perturbation theory 
has been proven to be very smooth [47, 48]. Thus eq. (3.3) provides us with a very economical model because one needs nothing but some infrared mass parameter which, being related to the gluon mass, is expected to be $\sim 1 \mathrm{GeV}$, to parametrize the deep infrared behaviour of the ghost-gluon transverse form factor. It should be noted that, although the ghost-gluon vertex form factors do not diverge, the gluon condensate does: it is the product of the Wilson coefficient and of the condensate which is expected to remain finite thanks to a delicate compensation of singularities, in the sum-rules approach. Then, both the Wilson coefficient and the condensate should be renormalized, by fixing some particular prescription, and both shall depend on a chosen renormalization momentum. This is explained in detail in the work of ref. [49] where the Wilson coefficient for the quark propagator is included at order $\mathcal{O}\left(\alpha^{4}\right)$. Since we carry out the computation of the Wilson coefficient in eqs. (2.4)-(2.9) only at tree-level and neglect its logarithmic dependence, we do not specify any renormalization momentum for the gluon condensate.

\subsection{Comparing with available lattice data}

Some $\mathrm{SU}(2)$ and $\mathrm{SU}(3)$ lattice results for the ghost-gluon vertex are available in the literature (see for instance ref. $[45,46]$ ). In particular, Cucchieri et al. have computed the tree-level-tensor form factor in $\mathrm{SU}(2)$ for three kinematics $(p \equiv q-k$ is the gluon momentum and $\varphi$ the angle between the gluon and ghost momenta): (1) $p^{2}=q^{2}$ and $\varphi=\pi / 2$, (2) $p^{2}=0$ and (3) $\varphi=\pi / 3$ with $p^{2}=q^{2}=k^{2}([45])$. As can be seen in the left pane of figure 1 , when we plug $g^{2}\left\langle A^{2}\right\rangle=6 \mathrm{GeV}^{2}, m_{\mathrm{IR}}=1.4 \mathrm{GeV}$ and $\widetilde{Z}_{1}^{-1}=1.04$ into eq. (3.3), the prediction for the form factor $H_{1}$ appears to agree pretty well with the $\mathrm{SU}(2)$ lattice data for the three kinematical configurations above mentioned. It should be also noted that the OPE prediction without the infrared completion through the introduction of $m_{\mathrm{IR}}$ given by eqs. (2.11), (2.14), plotted with dotted lines, also accounts very well for lattice data in the intermediate momenta region, above roughly $2.5 \mathrm{GeV}$. Furthermore, the value for the gluon condensate lies in the same ballpark as the estimates ${ }^{6}$ obtained from the $\mathrm{SU}(3)$ analysis of the Taylor coupling in ref. [26]. This strongly supports that the OPE analysis indeed captures the kinematical structure for the form factor $H_{1}$. On the other hand, the infrared mass scale, as supposed, is of the order of $1 \mathrm{GeV}$ and the (very close to 1 ) value for $\widetilde{Z}_{1}^{-1}$ accounts reasonably for perturbative value of $H_{1}$ that we approximated by a constant. Thus, after paying the economical price of incorporating only these two more parameters, both taking also reasonable values, we are left with a reliable closed formula for the form factor at any gluon or ghost momenta. This is a very useful ingredient for the numerical integration of the GPDSE.

The SU(3) results published by the authors of ref. [46] appear to be very noisy and cannot be invoked to properly discriminate whether a constant behaviour close to 1 or eq. (3.3) accounts better for them. However, we can now take the mass parameter, $m_{\mathrm{IR}}$, from the previous $\mathrm{SU}(2)$ analysis and the well-known $\mathrm{SU}(3)$ value for $g^{2}\left\langle A^{2}\right\rangle$, assume $\widetilde{Z}_{1}^{-1}=1$ and use eq. (3.3) to predict the ghost-gluon transverse form factor, $H_{1}$. This is

\footnotetext{
${ }^{6}$ In ref. [26], $g^{2}\left\langle A^{2}\right\rangle$ is evaluated through an OPE formula with a tree-level Wilson coefficient to be $5.1 \mathrm{GeV}^{2}$ at a renormalization point of $10 \mathrm{GeV}$.
} 

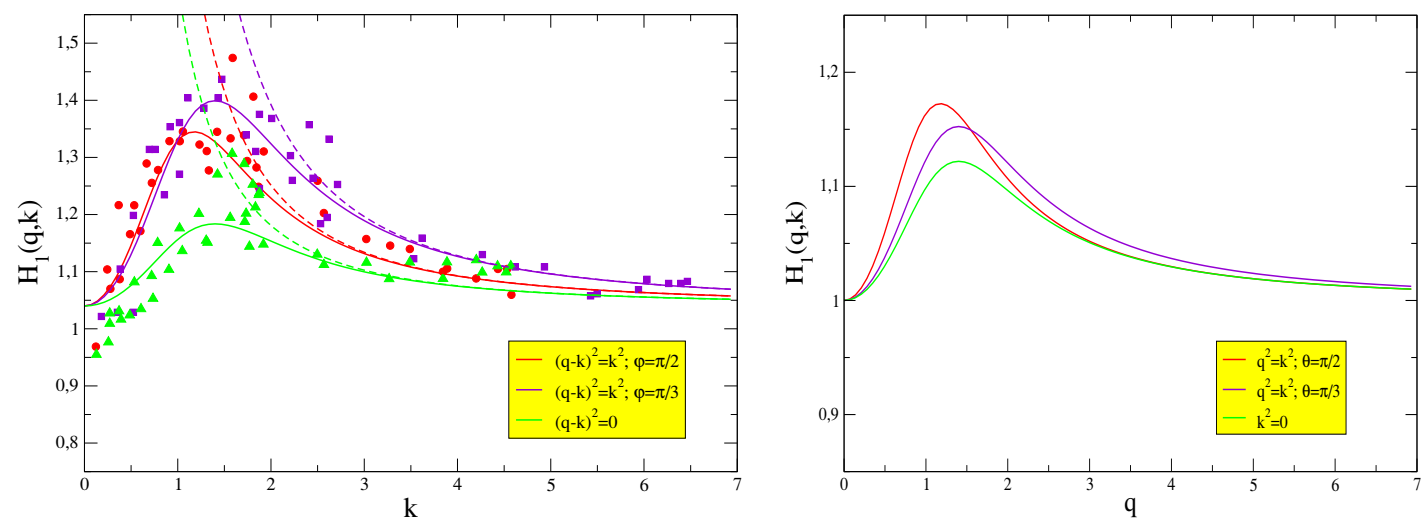

Figure 1. (Left.) The predictions of the model for $H_{1}$ (solid lines) in eq. (3.3) and the OPE result without infrared completion (dotted lines) in eq. (2.14) confronted to the $\mathrm{SU}(2)$ lattice data borrowed from ref. [45] for the three kinematical configurations described in the main text: $(k-q)^{2}=$ $q^{2}$ for $\varphi=\pi / 3$ (violet) and $\varphi=\pi / 2$ (red) and $(k-q)^{2}=0$ (green). (Right.) The results of the model for $H_{1}$ in the SU(3) case and for the following kinematical configurations: $k^{2}=q^{2}$ for $\theta=\pi / 3$ (violet) and $\theta=\pi / 2$ (red) and $k^{2}=0$ (green).

shown in the right plot of in figure 1 for three different kinematical configurations and, as can be seen, the deviations from 1 appear to be very small in all the cases and compatible with the results shown in figure 4 of ref. [46] for the vanishing gluon momentum case.

\section{The Taylor kinematics and the asymmetric gluon-ghost vertex}

An especially interesting kinematical configuration in the ghost-gluon vertex is the one where the incoming ghost momentum goes to zero $(k \rightarrow 0)$. Let us pay some additional attention to it. This particular kinematical limit is the one where Taylor's nonrenormalization theorem provides us with a result for the bare vertex, which is at least exact up to all perturbative ${ }^{7}$ orders:

$$
\Gamma_{\mathrm{bare}, \mu}^{a b c}(-q, 0 ; q)=-g f^{a b c} q_{\mu} .
$$

At any order of perturbation theory, this implies that $H_{1}(q, 0)+H_{2}(q, 0)=1$. According to eq. (3.3), one would have

$$
H_{1}(q, 0)=\widetilde{Z}_{1}^{-1}\left(1+N_{C} \frac{g^{2}\left\langle A^{2}\right\rangle}{4\left(N_{C}^{2}-1\right)} \frac{q^{2}}{q^{4}+m_{I R}^{4}}\right) .
$$

An interesting question to investigate is whether such a genuine non-perturbative correction still survives for the full ghost-gluon vertex in the Taylor limit, and not only for the

\footnotetext{
${ }^{7}$ The same argument of Taylor's perturbative proof still works if one consider the Landau-gauge ghostgluon vertex DSE: a vanishing ghost momentum entering in the vertex implies the contraction of the gluon-momentum transversal projector with the gluon momentum itself for any dressed diagram. Thus, Taylor's theorem is still in order within the non-perturbative DSE framework. Some attention have been recently paid to the ghost-gluon vertex DSE [50].
} 
transverse form factor $H_{1}$. Said otherwise, does eq.(4.1) maintains its validity upon adding the OPE corrections related to $\left\langle A^{2}\right\rangle$ to it, or not?

In order to properly address this question, one does not to have to study the ghostgluon vertex for asymptotically large legs' momenta, as done in section 2 , but to study instead the asymmetric ghost-gluon vertex

$$
\begin{aligned}
\widetilde{V}_{\mu}^{a b c}(-q, \varepsilon ; q-\varepsilon) & =\Gamma_{\mu^{\prime}}^{a^{\prime} b^{\prime} c^{\prime}}(-q, \varepsilon ; q-\varepsilon) G_{\mu \mu^{\prime}}^{b b^{\prime}}(q-\varepsilon) F^{a a^{\prime}}(q) F^{c c^{\prime}}(\varepsilon) \\
& =\int d^{4} x e^{i(q-\varepsilon) \cdot x}\left\langle T\left(\widetilde{c}^{c}(\varepsilon) A_{\mu}^{b}(x) \bar{c}^{a}(0)\right)\right\rangle
\end{aligned}
$$

where, when taking the limit $\varepsilon^{2} \rightarrow 0, \widetilde{c}^{a}(\varepsilon)$ stands for an incoming vanishing ghost field. A certain care is needed since we have to deal here with a "soft" ghost leg, similarly to the situation in ref. [40] when analyzing the three-gluon vertex. According to this, the key point for the OPE analysis of the asymmetric vertex is to apply the OPE expansion only to the fields carrying hard momenta and take then advantage of the operator expressions to compute the appropriate matrix elements in presence of a ghost field with "soft" momentum. Thus, we can apply to the asymmetric ghost-gluon vertex a procedure similar to the one outlined in section 2 and obtain

$$
\begin{aligned}
\widetilde{V}_{\mu}^{a b c}(-q, \varepsilon ; q-\varepsilon)= & \int d^{4} x e^{i(q-\varepsilon) \cdot x}\left\langle\widetilde{c}^{c}(\varepsilon) T\left(A_{\mu}^{b}(x) \bar{c}^{a}(0)\right)\right\rangle \\
= & \left(d_{1}\right)_{\mu c^{\prime}}^{a b}(q)\left\langle\widetilde{c}^{c}(\varepsilon): \bar{c}^{c^{\prime}}(0):\right\rangle \\
& +\left(d_{3}\right)_{\mu c^{\prime} a^{\prime} b^{\prime}}^{a b \nu^{\prime}}(q)\left\langle\widetilde{c}^{c}(\varepsilon): \bar{c}^{c^{\prime}}(0) A_{\mu^{\prime}}^{a^{\prime}}(0) A_{\nu^{\prime}}^{b^{\prime}}(0):\right\rangle+\cdots
\end{aligned}
$$

Then, if one follows standard SVZ techniques to obtain the perturbative expansion of OPE Wilson coefficients, we will take the perturbative vacuum for the matrix elements of eq. (4.4) and obtain:

$$
\widetilde{V}_{\text {pert }, \mu}^{a b c}(-q, \varepsilon ; q-\varepsilon)=\left(d_{1}\right)_{\mu c^{\prime}}^{a b}(q, \varepsilon) F^{c c^{\prime}}(\varepsilon),
$$

where $d_{1}$ can be straightforwardly identified with the perturbative expansion of the ghostgluon Green function, $\widetilde{V}$, with an amputated soft-ghost leg. Beyond this purely perturbative first contribution, we will take the matrix element of eq. (4.4) with two external soft gluons in the perturbative vacuum:

$$
\begin{aligned}
& \int d^{4} x e^{i(q-\varepsilon) \cdot x}\left\langle\widetilde{c}^{c}(\varepsilon) \widetilde{A}_{\mu^{\prime \prime}}^{a^{\prime \prime}}(0) \widetilde{A}_{\nu^{\prime \prime}}^{b^{\prime \prime}}(0) T\left(A_{\mu}^{b}(x) \bar{c}^{a}(y)\right)\right\rangle \\
& =\left(d_{3}\right)_{\mu c^{\prime} a^{\prime} b^{\prime}}^{a b \mu^{\prime} \nu^{\prime}}(q, \varepsilon)\left\langle 0\left|\widetilde{c}^{c}(\varepsilon) \widetilde{A}_{\mu^{\prime \prime}}^{a^{\prime \prime}}(0) \widetilde{A}_{\nu^{\prime \prime}}^{b^{\prime \prime}}(0): \bar{c}^{c^{\prime}}(0) A_{\mu^{\prime}}^{a^{\prime}}(0) A_{\nu^{\prime}}^{b^{\prime}}(0):\right| 0\right\rangle \\
& =\left(d_{3}\right)_{\mu c^{\prime} a^{\prime} b^{\prime}}^{a b \mu^{\prime} \nu^{\prime}}(q, \varepsilon) F^{c c^{\prime}}(\varepsilon)\left(G_{\mu^{\prime \prime} \mu^{\prime}}^{a^{\prime \prime} a^{\prime}}(0) G_{\nu^{\prime \prime} \nu^{\prime}}^{b^{\prime \prime} b^{\prime}}(0)+G_{\nu^{\prime \prime} \mu^{\prime}}^{b^{\prime \prime} a^{\prime}}(0) G_{\mu^{\prime \prime} \nu^{\prime}}^{a^{\prime \prime} b^{\prime}}(0)\right) .
\end{aligned}
$$

On the other hand, when considering a non-perturbative vacuum for the matrix elements of the local operators in the OPE expansion of eq. (4.4) and for a vanishing incoming ghost momentum $\varepsilon^{2}$, we will apply the factorization approximation (shown to work in ref. [40]) that follows:

$$
\begin{aligned}
\left\langle\widetilde{c}^{c}(\varepsilon): \bar{c}^{c^{\prime}}(0) A_{\mu^{\prime}}^{a^{\prime}}(0) A_{\nu^{\prime}}^{b^{\prime}}(0):\right\rangle & \rightarrow\left\langle 0\left|\widetilde{c}^{c}(\varepsilon) \bar{c}^{c^{\prime}}(0)\right| 0\right\rangle\left\langle: A_{\mu^{\prime}}^{a^{\prime}}(0) A_{\nu^{\prime}}^{b^{\prime}}(0):\right\rangle \\
& =F^{c c^{\prime}}(\varepsilon) \frac{\left\langle A^{2}\right\rangle}{4\left(N_{C}^{2}-1\right)} \delta^{a^{\prime} b^{\prime}} g_{\mu^{\prime} \nu^{\prime}} .
\end{aligned}
$$


Thus, eq. (4.4) can be written as follows:

$$
\begin{aligned}
\widetilde{V}_{\mu}^{a b c}(-q, \varepsilon ; q-\varepsilon)= & \widetilde{V}_{\mathrm{pert}, \mu}^{a b c}(-q, \varepsilon ; q-\varepsilon) \\
& +\underbrace{\left(d_{3}\right)_{\mu c^{\prime} a^{\prime} b^{\prime}}^{a b \mu^{\prime}}(q, \varepsilon) \delta^{a^{\prime} b^{\prime}} g_{\mu^{\prime} \nu^{\prime}}}_{\widetilde{w}_{\mu}^{a b c^{\prime}}} \frac{\left\langle A^{2}\right\rangle}{4\left(N_{C}^{2}-1\right)} F^{c c^{\prime}}(\varepsilon)+\cdots
\end{aligned}
$$

where, now from eq. (4.6),

$$
\widetilde{w}_{\mu}^{a b c^{\prime}}(q)=\frac{1}{2} \frac{\int d^{4} x e^{i q \cdot(x-y)}\left\langle\widetilde{c}^{c}(\varepsilon) \widetilde{A}_{\mu^{\prime \prime}}^{a^{\prime \prime}}(0) \widetilde{A}_{\nu^{\prime \prime}}^{b^{\prime \prime}}(0) T\left(A_{\mu}^{b}(x) \bar{c}^{a}(y)\right)\right\rangle}{G_{\mu^{\prime} \mu^{\prime \prime}}^{b^{\prime \prime}}(0) G_{\nu^{\prime} \nu^{\prime \prime}}^{a^{\prime} a^{\prime \prime}}(0) F^{c c^{\prime}}(\varepsilon)} \delta^{a^{\prime} b^{\prime}} g_{\mu^{\prime} \nu^{\prime}}
$$

However, in the Landau gauge, the transverse incoming gluon kills the longitudinal contribution for the ghost-gluon vertex and only the transverse form factor, $H_{1}$, survives. One needs then to compute the proper (1PI) ghost-gluon vertex, which can be formally writen as

$$
\Gamma_{\mu}^{a b c}(-q, k ; q-k)=\frac{V_{\mu^{\prime}}^{a^{\prime} b^{\prime} c^{\prime}}(-q, k ; q-k)}{G_{\mu \mu^{\prime}}^{b b^{\prime}}(q-k) F^{a a^{\prime}}(q) F^{c c^{\prime}}(k)} .
$$

It is clear that, because of eq. (4.5), one can factor the vanishing ghost propagator out in the r.h.s. of eq. (4.8) and this implies that $\widetilde{w}_{\mu}^{a b c^{\prime}}$ in eq. (4.9) directly provides with the correction for the ghost-gluon vertex with the vanishing "soft" ghost leg explicitly amputated. Thus, we can formally define

$$
\widetilde{w}_{\mu}^{a b c^{\prime}}(q)=\widetilde{v}_{\mu^{\prime}}^{a^{\prime} b^{\prime} c^{\prime}}(q) F^{a^{\prime} a}(q) G_{\mu^{\prime} \mu}^{b^{\prime} b}(q)
$$

such that the proper ghost-gluon vertex can be obtained as

$$
\Gamma_{\mu}^{a b c}(-q, \varepsilon ; q-\varepsilon)=\Gamma_{\text {pert }, \mu}^{a b c}(-q, \varepsilon ; q-\varepsilon)+\widetilde{v}_{\mu}^{a b c} \frac{\left\langle A^{2}\right\rangle}{4\left(N_{C}^{2}-1\right)}+\cdots
$$

where

$$
\widetilde{v}_{\mu}^{a b c}=2 I^{[1]},
$$

the factor 2 coming from the combinatorics and $I^{[1]}$ being the following diagram (no external legs)

$$
\begin{aligned}
& I^{[1]}={ }_{-\varepsilon}^{q-\varepsilon} g_{-\rightarrow--\xi_{-q}} \\
& =-\frac{N_{C}}{2} g^{3} \frac{(q-\varepsilon)_{\sigma} q_{\sigma}}{q^{2}(q-\varepsilon)^{2}} f^{a b c}\left(q_{\mu}-\frac{q_{\mu_{2}}(q-\varepsilon)_{\mu_{2}}}{(q-\varepsilon)^{2}}(q-\varepsilon)_{\mu}\right) .
\end{aligned}
$$


Then, according to eq. (1.2), one finally obtains:

$$
\Gamma_{\mu}^{a b c}(-q, \varepsilon ; q-\varepsilon)=-g f^{a b c}\left(q_{\mu} H_{1}(q, \varepsilon)+(q-\varepsilon)_{\mu} H_{2}(q, \varepsilon)\right)+\cdots
$$

where

$$
\begin{aligned}
& H_{1}(q, \varepsilon)=H_{1}^{\text {pert }}(q, \varepsilon)+N_{C} g^{2} \frac{(q-\varepsilon) \cdot q}{q^{2}(q-\varepsilon)^{2}} \frac{\left\langle A^{2}\right\rangle}{4\left(N_{C}^{2}-1\right)}, \\
& H_{2}(q, \varepsilon)=H_{2}^{\text {pert }}(q, \varepsilon)-N_{C} g^{2} \frac{((q-\varepsilon) \cdot q)^{2}}{q^{2}(q-\varepsilon)^{4}} \frac{\left\langle A^{2}\right\rangle}{4\left(N_{C}^{2}-1\right)} .
\end{aligned}
$$

Thus, after taking the limit $\varepsilon \rightarrow 0$, one will have:

$$
\Gamma_{\mu}^{a b c}(-q, 0 ; q)=-g f^{a b c} q_{\mu}\left(H_{1}^{\mathrm{pert}}(q, 0)+H_{2}^{\mathrm{pert}}(q, 0)\right)=-g f^{a b c} q_{\mu} .
$$

This confirms that no non-perturbative OPE correction survives in the proper ghost-gluon vertex in the Taylor limit, although both form factors $H_{1}$ and $H_{2}$ separately undergo such a kind of correction. It is also straightforward to see that $H_{1}(q, 0)$ obtained from eq. (4.16) would be the same as the one given in eq. (4.2), obtained for the general kinematics, when $q^{2} \gg m_{\mathrm{IR}}^{2}$. Furthermore, provided we introduce in eq. (4.16) for $H_{1}$ the same infrared mass scale, $m_{I R}$, we used to obtain eq. (4.2) we would again be left with the same result. These observations underline a rewarding agreement of our resuts although they stem from slightly different hypotheses.

\section{Conclusions}

The momentum behaviour of the ghost-gluon vertex has been studied in the framework of the operator product expansion (OPE). This approach has already been proved in the literature to be very fruitful in describing the running of two-point gluon and quark Green functions and of the strong coupling computed in several MOM renormalization schemes. In particular, the coupling derived from the ghost-gluon vertex renormalized in T-scheme, which can be directly computed from nothing else but the ghost and gluon propagators, led to a very accurate determination of $\Lambda_{\mathrm{QCD}}$ when its running with momenta obtained from the lattice estimate has been confronted with the OPE prediction, although only after accounting properly for a dimension-two gluon condensate, $\left\langle A^{2}\right\rangle$. In this note, we followed the same approach to study the ghost-gluon vertex and payed special attention to the transverse form factor for the ghost-gluon vertex, precisely the one that plays a crucial rôle for the truncation and resolution of the GPDSE and that, in this context, is usually approximated by a constant. In this way we obtained a genuine non-perturbative OPE correction to the perturbative part for the transverse form factor. A very simple conjecture was then made to extend the OPE description beyond the momentum range where the SVZ factorization is supposed to work and we were left with a simple model describing the momentum behaviour of the ghost-gluon form factor. This model was shown to agree pretty well with a lattice $\mathrm{SU}(2)$ computation for several kinematical configurations 
of the ghost-gluon vertex, when the value for the gluon condensate is in the same ballpark as the one that is estimated from the running of the T-scheme coupling in $\mathrm{SU}(3)$ lattice gauge theory. This successful comparison with the available lattice data provides us with strong indications that (i) the OPE framework helps to account for the kinematical structure of the ghost-gluon vertex at intermediate momentum domain and (ii) that it helps to cook up a reliable model, providing a good parameterization also for the IR domain, to be plugged into the GPDSE to reproduce the ghost propagator lattice data. These are the main goals of the paper.

Finally, we also proved that, in the particular kinematical limit associated with the Taylor theorem and hence by the T-scheme (a vanishing incoming ghost momentum), the corrections to the longitudinal and transverse form factors cancel against each other and that the tree-level result for the ghost-gluon vertex is thus recovered.

In future work, we foresee to put to use the vertex (3.3) in combination with the GPDSE, this in order to investigate at the quantitative level how this vertex allows to bring the analytical predictions of the GPDSE in closer agreement with the lattice output for the ghost propagator, given the input of the kernel via the lattice gluon propagator.

\section{Acknowledgments}

J. R-Q acknowledges the Spanish MICINN for the support by the research project FPA200910773 and "Junta de Andalucia" by P07FQM02962. D. Dudal is supported by the ResearchFoundation Flanders (FWO Vlaanderen). We thank A. Le Yaouanc, J. Micheli and A. Cucchieri for inspiring discussions, while J. R-Q acknowledges A. C. Aguilar and J. Papavassiliou for very valuable indications triggering the work.

Open Access. This article is distributed under the terms of the Creative Commons Attribution Noncommercial License which permits any noncommercial use, distribution, and reproduction in any medium, provided the original author(s) and source are credited.

\section{References}

[1] A. Cucchieri and T. Mendes, Constraints on the IR behavior of the gluon propagator in Yang-Mills theories, Phys. Rev. Lett. 100 (2008) 241601 [arXiv:0712.3517] [INSPIRE].

[2] A. Cucchieri and T. Mendes, Constraints on the IR behavior of the ghost propagator in Yang-Mills theories, Phys. Rev. D 78 (2008) 094503 [arXiv:0804.2371] [INSPIRE].

[3] A. Cucchieri and T. Mendes, Landau-gauge propagators in Yang-Mills theories at $\beta=0$ : massive solution versus conformal scaling, Phys. Rev. D 81 (2010) 016005 [arXiv: 0904.4033] [INSPIRE].

[4] P. Costa, O. Oliveira and P. Silva, What does low energy physics tell us about the zero momentum gluon propagator, Phys. Lett. B 695 (2011) 454 [arXiv:1011.5603] [INSPIRE].

[5] O. Oliveira and P. Bicudo, Running gluon mass from Landau gauge lattice QCD propagator, J. Phys. G 38 (2011) 045003 [arXiv: 1002.4151] [inSPIRE]. 
[6] I. Bogolubsky, V. Bornyakov, G. Burgio, E. Ilgenfritz, M. Muller-Preussker, et al., Improved Landau gauge fixing and the suppression of finite-volume effects of the lattice gluon propagator, Phys. Rev. D 77 (2008) 014504 [Erratum ibid. D 77 (2008) 039902] [arXiv:0707.3611] [INSPIRE].

[7] I. Bogolubsky, E. Ilgenfritz, M. Muller-Preussker and A. Sternbeck, Lattice gluodynamics computation of Landau gauge Green's functions in the deep infrared, Phys. Lett. B 676 (2009) 69 [arXiv:0901.0736] [INSPIRE].

[8] V. Bornyakov, V. Mitrjushkin and M. Muller-Preussker, SU(2) lattice gluon propagator: continuum limit, finite-volume effects and infrared mass scale $m_{\mathrm{IR}}$, Phys. Rev. D 81 (2010) 054503 [arXiv:0912.4475] [inSPIRE].

[9] A. Aguilar, D. Binosi and J. Papavassiliou, Gluon and ghost propagators in the Landau gauge: deriving lattice results from Schwinger-Dyson equations, Phys. Rev. D 78 (2008) 025010 [arXiv:0802.1870] [inSPIRE].

[10] D. Binosi and J. Papavassiliou, Pinch technique: theory and applications, Phys. Rept. 479 (2009) 1 [arXiv:0909.2536] [InSPIRE].

[11] P. Boucaud, J. Leroy, A. Le Yaouanc, J. Micheli, O. Pène, et al., On the IR behaviour of the Landau-gauge ghost propagator, JHEP 06 (2008) 099 [arXiv: 0803.2161] [INSPIRE].

[12] P. Boucaud, J.-P. Leroy, A. Yaouanc, J. Micheli, O. Pène, et al., IR finiteness of the ghost dressing function from numerical resolution of the ghost SD equation, JHEP 06 (2008) 012 [arXiv:0801.2721] [INSPIRE].

[13] P. Boucaud, M. Gomez, J. Leroy, A. Le Yaouanc, J. Micheli, et al., The low-momentum ghost dressing function and the gluon mass, Phys. Rev. D 82 (2010) 054007 [arXiv:1004.4135] [INSPIRE].

[14] J. Rodríguez-Quintero, On the massive gluon propagator, the PT-BFM scheme and the low-momentum behaviour of decoupling and scaling DSE solutions, JHEP 01 (2011) 105 [arXiv: 1005.4598] [INSPIRE].

[15] C.S. Fischer, A. Maas and J.M. Pawlowski, On the infrared behavior of Landau gauge Yang-Mills theory, Annals Phys. 324 (2009) 2408 [arXiv:0810.1987] [INSPIRE].

[16] C.S. Fischer and J.M. Pawlowski, Uniqueness of infrared asymptotics in Landau gauge Yang-Mills theory II, Phys. Rev. D 80 (2009) 025023 [arXiv: 0903.2193] [inSPIRE].

[17] R. Alkofer, M.Q. Huber and K. Schwenzer, Infrared singularities in Landau gauge Yang-Mills theory, Phys. Rev. D 81 (2010) 105010 [arXiv:0801.2762] [INSPIRE].

[18] D. Dudal, R. Sobreiro, S. Sorella and H. Verschelde, The Gribov parameter and the dimension two gluon condensate in Euclidean Yang-Mills theories in the Landau gauge, Phys. Rev. D 72 (2005) 014016 [hep-th/0502183] [INSPIRE].

[19] D. Dudal, S. Sorella, N. Vandersickel and H. Verschelde, New features of the gluon and ghost propagator in the infrared region from the Gribov-Zwanziger approach, Phys. Rev. D 77 (2008) 071501 [arXiv:0711.4496] [INSPIRE].

[20] D. Dudal, J.A. Gracey, S.P. Sorella, N. Vandersickel and H. Verschelde, A refinement of the Gribov-Zwanziger approach in the Landau gauge: infrared propagators in harmony with the lattice results, Phys. Rev. D 78 (2008) 065047 [arXiv: 0806.4348] [INSPIRE]. 
[21] D. Dudal, O. Oliveira and N. Vandersickel, Indirect lattice evidence for the refined Gribov-Zwanziger formalism and the gluon condensate $\left\langle A^{2}\right\rangle$ in the Landau gauge, Phys. Rev. D 81 (2010) 074505 [arXiv: 1002 . 2374] [INSPIRE].

[22] M. Tissier and N. Wschebor, Infrared propagators of Yang-Mills theory from perturbation theory, Phys. Rev. D 82 (2010) 101701 [arXiv:1004.1607] [INSPIRE].

[23] M. Tissier and N. Wschebor, An infrared safe perturbative approach to Yang-Mills correlators, Phys. Rev. D 84 (2011) 045018 [arXiv: 1105.2475] [INSPIRE].

[24] M. Pennington and D. Wilson, Are the dressed gluon and ghost propagators in the Landau gauge presently determined in the confinement regime of QCD?, arXiv:1109.2117 [INSPIRE].

[25] J. Taylor, Ward identities and charge renormalization of the Yang-Mills field, Nucl. Phys. B 33 (1971) 436 [InSPIRE].

[26] P. Boucaud, F. De Soto, J. Leroy, A. Le Yaouanc, J. Micheli, et al., Ghost-gluon running coupling, power corrections and the determination of $\Lambda_{M \bar{S}}$, Phys. Rev. D 79 (2009) 014508 [arXiv:0811.2059] [INSPIRE].

[27] ETM collaboration, B. Blossier et al., Ghost-gluon coupling, power corrections and $\Lambda_{\overline{\mathrm{MS}}}$ from twisted-mass lattice QCD at $N_{f}=2$, Phys. Rev. D 82 (2010) 034510 [arXiv:1005.5290] [INSPIRE].

[28] A. Sternbeck, E.-M. Ilgenfritz, K. Maltman, M. Muller-Preussker, L. von Smekal, et al., QCD $\lambda$ parameter from Landau-gauge gluon and ghost correlations, PoS LAT2009 (2009) 210 [arXiv: 1003.1585] [INSPIRE].

[29] A. Aguilar, D. Binosi, J. Papavassiliou and J. Rodríguez-Quintero, Non-perturbative comparison of QCD effective charges, Phys. Rev. D 80 (2009) 085018 [arXiv:0906.2633] [INSPIRE].

[30] P. Boucaud, J. Leroy, A. Yaouanc, J. Micheli, O. Pène, et al., The infrared behaviour of the pure Yang-Mills Green functions, arXiv:1109.1936 [INSPIRE].

[31] J. Rodríguez-Quintero, On the gluon condensat, the ghost-gluon vertex and the Taylor theorem, Proceedings of TNT-2011, September 5-9, Trento Italy (2011).

[32] D. Dudal, Ghost dissection, Proceedings of TNT-2011, September 5-9, Trento Italy (2011).

[33] B. Alles, D. Henty, H. Panagopoulos, C. Parrinello, C. Pittori, et al., $\alpha_{s}$ from the nonperturbatively renormalised lattice three gluon vertex, Nucl. Phys. B 502 (1997) 325 [hep-lat/9605033] [INSPIRE].

[34] D. Becirevic, P. Boucaud, J. Leroy, J. Micheli, O. Pène, et al., Asymptotic behavior of the gluon propagator from lattice QCD, Phys. Rev. D 60 (1999) 094509 [hep-ph/9903364] [INSPIRE].

[35] D. Becirevic, P. Boucaud, J. Leroy, J. Micheli, O. Pène, et al., Asymptotic scaling of the gluon propagator on the lattice, Phys. Rev. D 61 (2000) 114508 [hep-ph/9910204] [INSPIRE].

[36] P. Boucaud, G. Burgio, F. Di Renzo, J. Leroy, J. Micheli, et al., Lattice calculation of $1 / p^{2}$ corrections to $\alpha_{s}$ and of $\Lambda_{\mathrm{QCD}}$ in the MOM scheme, JHEP 04 (2000) 006 [hep-ph/0003020] [INSPIRE].

[37] P. Boucaud, A. Le Yaouanc, J. Leroy, J. Micheli, O. Pène, et al., Consistent OPE description of gluon two point and three point Green function?, Phys. Lett. B 493 (2000) 315 [hep-ph/0008043] [INSPIRE]. 
[38] P. Boucaud, J. Leroy, H. Moutarde, J. Micheli, O. Pène, et al., Preliminary calculation of $\alpha_{s}$ from Green functions with dynamical quarks, JHEP 01 (2002) 046 [hep-ph/0107278] [INSPIRE].

[39] P. Boucaud, A. Le Yaouanc, J. Leroy, J. Micheli, O. Pène, et al., Testing Landau gauge OPE on the lattice with a $\left\langle A^{2}\right\rangle$ condensate, Phys. Rev. D 63 (2001) 114003 [hep-ph/0101302] [INSPIRE].

[40] F. De Soto and J. Rodríguez-Quintero, Notes on the determination of the Landau gauge OPE for the asymmetric three gluon vertex, Phys. Rev. D 64 (2001) 114003 [hep-ph/0105063] [INSPIRE].

[41] P. Boucaud, J. Leroy, A. Le Yaouanc, A. Lokhov, J. Micheli, et al., Non-perturbative power corrections to ghost and gluon propagators, JHEP 01 (2006) 037 [hep-lat/0507005] [INSPIRE].

[42] F.D. Bonnet, P.O. Bowman, D.B. Leinweber, A.G. Williams and J.M. Zanotti, Infinite volume and continuum limits of the Landau gauge gluon propagator, Phys. Rev. D 64 (2001) 034501 [hep-lat/0101013] [INSPIRE].

[43] F.D. Bonnet, P.O. Bowman, D.B. Leinweber and A.G. Williams, Infrared behavior of the gluon propagator on a large volume lattice, Phys. Rev. D 62 (2000) 051501 [hep-lat/0002020] [INSPIRE].

[44] A.G. Williams, P. Bowman, F. Bonnet, D. Leinweber, and J. Skullerud, Gluon and quark propagators in Landau gauge from the lattice, proceedings of the Workshop on Lightcone QCD and Nonperturbative Hadron Physics, December 13-22 1999, Adelaide Australia, A.W. Schreiber and A.G. Williams eds., World Scientific, Singapore (1999).

[45] A. Cucchieri, A. Maas and T. Mendes, Three-point vertices in Landau-gauge Yang-Mills theory, Phys. Rev. D 77 (2008) 094510 [arXiv: 0803.1798] [INSPIRE].

[46] A. Sternbeck, E.-M. Ilgenfritz, M. Muller-Preussker and A. Schiller, Landau gauge ghost and gluon propagators and the Faddeev-Popov operator spectrum, Nucl. Phys. Proc. Suppl. 153 (2006) 185 [hep-lat/0511053] [InSPIRE].

[47] K. Chetyrkin and A. Retey, Three loop three linear vertices and four loop similar to MOM $\beta$-functions in massless $Q C D$, hep-ph/0007088 [INSPIRE].

[48] A.I. Davydychev, P. Osland and O. Tarasov, Three gluon vertex in arbitrary gauge and dimension, Phys. Rev. D 54 (1996) 4087 [Erratum ibid. D 59 (1999) 109901] [hep-ph/9605348] [INSPIRE].

[49] B. Blossier, P. Boucaud, M. Brinet, F. De Soto, Z. Liu, et al., Renormalisation of quark propagators from twisted-mass lattice $Q C D$ at $N_{f}=2$, Phys. Rev. D 83 (2011) 074506 [arXiv: 1011.2414] [INSPIRE].

[50] W. Schleifenbaum, A. Maas, J. Wambach and R. Alkofer, Infrared behaviour of the ghost-gluon vertex in Landau gauge Yang-Mills theory, Phys. Rev. D 72 (2005) 014017 [hep-ph/0411052] [INSPIRE]. 